\title{
EFEITO DE UM PROGRAMA EDUCACIONAL EM EMPODERAMENTO DO AUTOCUIDADO PARA CUMPRIMENTO DE METAS EM DIABETES
}

\author{
EFFECTS OF AN EDUCATIONAL PROGRAM ON SELF-CARE \\ EMPOWERMENT FOR THE FULFILLMENT OF GOALS IN DIABETES
}

\section{EFECTO DE UN PROGRAMA EDUCATIVO EN EMPODERAMIENTO DEL AUTOCUIDADO PARA EL CUMPLIMIENTO DE OBJETIVOS EN DIABETES}

\author{
Daniel Nogueira Cortez ${ }^{*}$ \\ Jéssica Caroline dos Santos ** \\ Maísa Mara L. Macedo ${ }^{* * *}$ \\ Débora Aparecida Silva Souza ${ }^{* * *}$ \\ IlKa AFONSO ReIS ${ }^{* * * *}$ \\ Heloísa Carvalho Torres ${ }^{* * * * *}$
}

\section{RESUMO}

Objetivo: Avaliar o efeito de um programa educacional baseado no empoderamento das práticas de autocuidado para favorecer o cumprimento de metas em usuários com diabetes tipo 2 na Atenção Primária à Saúde. Material e método: Estudo quasi-experimental, do tipo antes e depois realizado durante 12 meses, com 127 usuários com diabetes mellitus tipo 2 em seguimento num programa educacional para empoderamento em práticas de autocuidado, em cinco unidades de saúde da família da atenção primária à saúde em uma cidade de Minas Gerais, Brasil. Para os dados sociodemográficos utilizou-se instrumento próprio criado para a pesquisa. Foram avaliadas as variáveis: características sociodemográficas colhidas por meio de entrevista e instrumento próprio criado para a pesquisa, características clínicas e metabólicas extraídos por meio de coleta sanguínea, nível de empoderamento colhido por meio da Escala de Empoderamento e cumprimento de metas por meio de entrevista e instrumento próprio criado para a pesquisa. Resultados: Entre os participantes, 33,9\% (43) dos usuários autodeclararam cumprirem parcialmente e $66,1 \%$ (84) totalmente as metas, para o favorecimento da mudança em comportamento e melhora do controle do diabetes. Em relação ao empoderamento, a chance de cumprimento total de metas no grupo com maior pontuação é 2,98 vezes a chance do grupo com menor pontuação (IC95\%: 1,30-6,86). Conclusão: Evidenciou-se que o programa educacional baseado no empoderamento das práticas de autocuidado favoreceu o cumprimento de metas em usuários com diabetes tipo 2 na Atenção Primária à Saúde.

\footnotetext{
* Doutor. Professor do curso de Enfermagem. Universidade Federal de São João del Rei, Campus Centro Oeste Dona Lindu. Divinópolis, Brasil. Email: danielcortez@ufsj.edu.br. Autor correspondente.

${ }^{* *}$ Doutoranda pela Escola de Enfermagem da Universidade Federal de Minas Gerais, Escola de Enfermagem. Belo Horizonte, Brasil. Email: jessica-caroline31@hotmail.com

${ }^{* * *}$ Mestre pela Escola de Enfermagem da Universidade Federal de Minas Gerais, Escola de Enfermagem. Belo Horizonte, Brasil. Email: maisamlm@hotmail.com

${ }^{* * * *}$ Mestre pela Escola de Enfermagem da Universidade Federal de Minas Gerais, Escola de Enfermagem. Belo Horizonte, Brasil. Email: deboraassenf@gmail.com

${ }_{* * * * *}$ Doutora. Professora do Departamento de Estatística. Universidade Federal de Minas Gerais, Departamento de Estatística. Belo Horizonte, Brasil. Email: ilka@est.ufmg.br

${ }^{* * * * * *}$ Pós-Doutora. Professora da Escola de Enfermagem Universidade Federal de Minas Gerais, Escola de Enfermagem. Belo Horizonte, Brasil. Email: heloisa@enf.ufmg.br
} 
Palavras chave: Autocuidado, Diabetes Mellitus tipo 2, participação do paciente, metas, planejamento de assistência ao paciente, Atenção Primária à Saúde.

\begin{abstract}
Objective: To evaluate the effect of an educational program based on the empowerment of self-care practices to favor the fulfillment of goals in users with type 2 diabetes in Primary Health Care. Methods: Pre-post quasi-experimental study, performed for 12 months with 127 users with type 2 diabetes mellitus, enrolled and followed up in an educational program for the empowerment of self-care practices. The following variables were evaluated: sociodemographic data collected through an interview and a specific instrument created for the research, clinical and metabolic characteristics collected by means of blood tests, degree of empowerment gathered through the Empowerment Scale, and goals fulfillment by means of interview and the specific instrument created for the research. Results: Among the participants, 33.9\% (43) of users self-reported partially meeting, and $66.1 \%$ (84) fully meeting the goals, favoring a behavior change and improving diabetes control. Regarding empowerment, the chance of achieving the total goals in the group with the highest score is 2.98 times that of the group with the lowest score (95\% CI: 1.30-6.86). Conclusion: It was evidenced that the educational program based on the empowerment of self-care practices favored the achievement of goals in users with type 2 diabetes in Primary Health Care.
\end{abstract}

Key words: Self-care, Diabetes Mellitus Type 2, patient participation, goals, patient care planning, primary healthcare.

\title{
RESUMEN
}

Objetivo: Evaluar el efecto de un programa educativo basado en el empoderamiento de prácticas de autocuidado para favorecer el cumplimiento de metas en usuarios con diabetes tipo 2 en Atención Primaria a la Salud. Material y método: Estudio cuasi experimental, del tipo antes y después, realizado durante 12 meses, con 127 usuarios con Diabetes Mellitus tipo 2 en seguimiento de un programa educativo para empoderamiento en prácticas de autocuidado. Se evaluaron las variables: características sociodemográficas recogidas por medio de una entrevista e instrumento propio creado para la investigación, clínicas y metabólicas recogidas por la recolección de sangre, empoderamiento recogida a través de la Escala de Empoderamiento y cumplimiento de objetivos por medio de una entrevista e instrumento propio creado para la investigación. Resultados: Entre los participantes, el 33,9\% (43) de los usuarios autodeclararon cumplir parcialmente y el 66,1\% (84) totalmente las metas para el favorecimiento del cambio de comportamiento y la mejora del control de la diabetes. Con respecto al empoderamiento, la posibilidad de cumplimiento total de metas en el grupo con mayor puntuación es 2,98 veces esa oportunidad en el grupo con menor puntuación (IC 95\%: 1,30-6,86). Conclusión: Se evidenció que el programa educativo basado en el empoderamiento de las prácticas de autocuidado favoreció el cumplimiento de metas en usuarios con diabetes tipo 2 para la Atención Primaria a la Salud.

Palabras clave: Autocuidado, Diabetes Mellitus tipo 2, participación del paciente, metas, planificación de atención al paciente, Atención Primaria de Salud.

Fecha recepción: 02/08/17 Fecha aceptación: 29/04/18 


\section{INTRODUÇÃO}

Na América Central e do Sul havia 26 milhões de casos de Diabetes Mellitus em 2017 e uma estimativa de 42,3 milhões para 2045. Apenas no Brasil havia mais de 12,5 milhões de casos em 2017 (1). No desenvolvimento do diabetes, destaca-se a alta morbimortalidade com a presença de complicações agudas e crônicas, além dos custos no controle e tratamento dessa condição $(2,3)$.

A situação do diabetes no Brasil e no mundo tem exigido dos gestores, profissionais da área da saúde e dos centros de pesquisas, a busca por abordagens que contemplem práticas inovadoras e uma mudança de paradigma que reconheça que os usuários precisam estar no controle do diabetes (4). Estudos têm demonstrado que o aumento da participação do usuário em responsabilizar-se pelo seu cuidado diário pode melhorar os comportamentos em relação à saúde, levando o usuário a adquirir autonomia na tomada de decisões para a mudança de comportamento e ser agente das transformações para o autocuidado (4-6).

As práticas baseadas no empoderamento, são apropriadas em usuários com Diabetes Mellitus tipo 2 e fundamentam-se em três aspectos: escolhas, controle e consequências (7). A abordagem do empoderamento é pautada, principalmente, na problematização para aquisição de conhecimentos, habilidades e atitudes para o autocuidado, enfatizando o problema e a resposta do usuário, sempre, com estímulo à reflexão para conclusões corretas, num processo de troca e cumprimento de metas para o autocuidado do diabetes (6). Parte-se do princípio que o usuário empoderado sobre seu cuidado é capaz de tomar decisões informadas de forma autônoma em seu próprio benefício, com habilidade de reconhecer e avaliar seu comportamento e definir metas reais que contribuam com as práticas de autocuidado $(8,9)$.
Destaca-se que o diabetes é uma condição crônica de saúde que engloba cuidados diários que são realizados pelo próprio usuário. Contudo, é importante incentivar e implementar intervenções educativas empoderadoras que estimulem a sua autonomia e ofereça apoio para cuidar de si. O empoderamento é resultado da educação em diabetes e este tipo de abordagem faz alusão ao processo pelo qual as pessoas ganham controle e domínio sobre suas próprias vidas, garantindo-lhes as condições para aumentar a motivação para realizar tarefas $(10,11)$.

No programa educativo esta abordagem foi utilizada visando desempenhar uma avaliação sobre o desenvolvimento de habilidades para práticas de autocuidado em busca de favorecer o cumprimento de metas para alcançar o controle glicêmico. É importante destacar que a construção e o cumprimento de metas são ações realizadas pelo usuário que o ajuda a resolver as dificuldades do cotidiano e alcançar seus objetivos.

Os resultados sobre a relação entre o empoderamento do usuário com diabetes e o controle metabólico é conflitante em alguns estudos, mas o desenvolvimento de programas educativos baseados no empoderamento pode ser uma alternativa no controle do diabetes $(7,8,12)$. A partir do programa educativo em diabetes, realizado em um município brasileiro, observou-se a decisão do usuário pelo cumprimento de metas para mudança de comportamento de forma a atingir hábitos de vida saudável.

Neste cenário, o enfermeiro como um dos membros da equipe da Atenção Primária à Saúde, atua de maneira contínua na prevenção e promoção da saúde dos usuários com diabetes tipo 2. Por meio da sua atuação, o profissional tem a possibilidade de realizar educação em saúde para o restabelecimento e a manutenção do controle glicémico $(13,14)$. Dentro desta perspectiva, realizar de estratégias educativas baseados no empoderamento pode ser uma ferramenta eficaz, 
viável, acessível e capaz de ajudar no monitoramento do autocuidado do usuário no seu cotidiano. Além disso, permite autoavaliar a sua capacidade no cumprimento de metas para fomentar uma vida mais saudável (11).

Assim, espera-se que o usuário do serviço de saúde que participa de um programa educativo embasado no empoderamento, consiga compreender os motivos de participar ativamente do seu tratamento, realizar práticas de autocuidado e cumprir metas para o controle do diabetes.

Neste sentido, o objetivo deste estudo foi avaliar o efeito de um programa educativo baseado no empoderamento para favorecer o cumprimento de metas em usuários com diabetes tipo 2 para as práticas de autocuidado na Atenção Primária à Saúde.

\section{MATERIAL E MÉTODO}

Tipo do estudo: Estudo quase-experimental do tipo antes e depois desenvolvido em cinco unidades de saúde da família da atenção primária de uma cidade de Minas Gerais, Brasil (15).

População: Baseado no cadastro municipal de registros de usuários com Diabetes e em estudo do grupo de pesquisa envolvendo a mesma população de estudo (16), foi calculado o tamanho da amostra considerando-se o efeito de cluster e os valores de Hemoglobina glicada (HbA1c) e escolaridade (17). Os critérios de inclusão foram: ter DM tipo 2 sem complicações, ter condições de realizar autocuidado. O estudo iniciou com 162 usuários e após perdas por mudança de endereço, morte e desinteresse em continuar no estudo, a amostra constituiu-se de 127 usuários.

Programa educativo: Partiu-se da hipótese que os usuários com maior pontuação para o empoderamento refeririam mais cumprimento de metas total em comparação com aqueles com menor pontuação para o empoderamento, além de diminuir a $\mathrm{HbAlc}$ ao final do programa educativo. Este programa foi estruturado seguindo o protocolo mudança de comportamento, já validado para o Brasil (10), dividido em cinco passos: 1) exploração do problema; 2) esclarecimento de sentimentos e significados; 3) desenvolvimento de um plano; 4) comprometimento com a ação e 5) avaliação da experiência e do plano. O programa ocorreu entre novembro de 2014 a janeiro de 2016, totalizando dez encontros com os usuários. Em cada encontro o usuário propunha uma meta a ser cumprida, que se relacionava à mudança de comportamento para o controle do diabetes. No encontro seguinte a meta era discutida, seguia-se o protocolo e mais uma nova meta era definida pelo usuário, incentivada pela discussão do grupo, e assim por diante. Junto ao protocolo, as metodologias de ensino aprendizagem utilizadas criavam estratégias para motivar e reforçar o autocuidado do diabetes (6). O programa educativo em diabetes, conduzido pelo enfermeiro, seguiu o seguinte modelo (Figura 1).

Coleta e análise dos dados: As variáveis avaliadas foram sociodemográficas, tempo de diagnóstico, presença de outras doenças, tipo de tratamento, HbA1c, cumprimento de metas e nível de empoderamento. O perfil dos usuários foi descrito em frequências relativas (\%) das variáveis categóricas sexo, escolaridade, ocupação e estado civil. Para as variáveis idade e tempo de diagnóstico, foram calculadas as estatísticas descritivas de tendência central e de variabilidade. A HbAlc inicial (antes da intervenção) e final (pós intervenção) foram comparadas para avaliação do programa educativo. 
Figura 1. Modelo das etapas do programa de empoderamento para autocuidado em diabetes Mellitus.

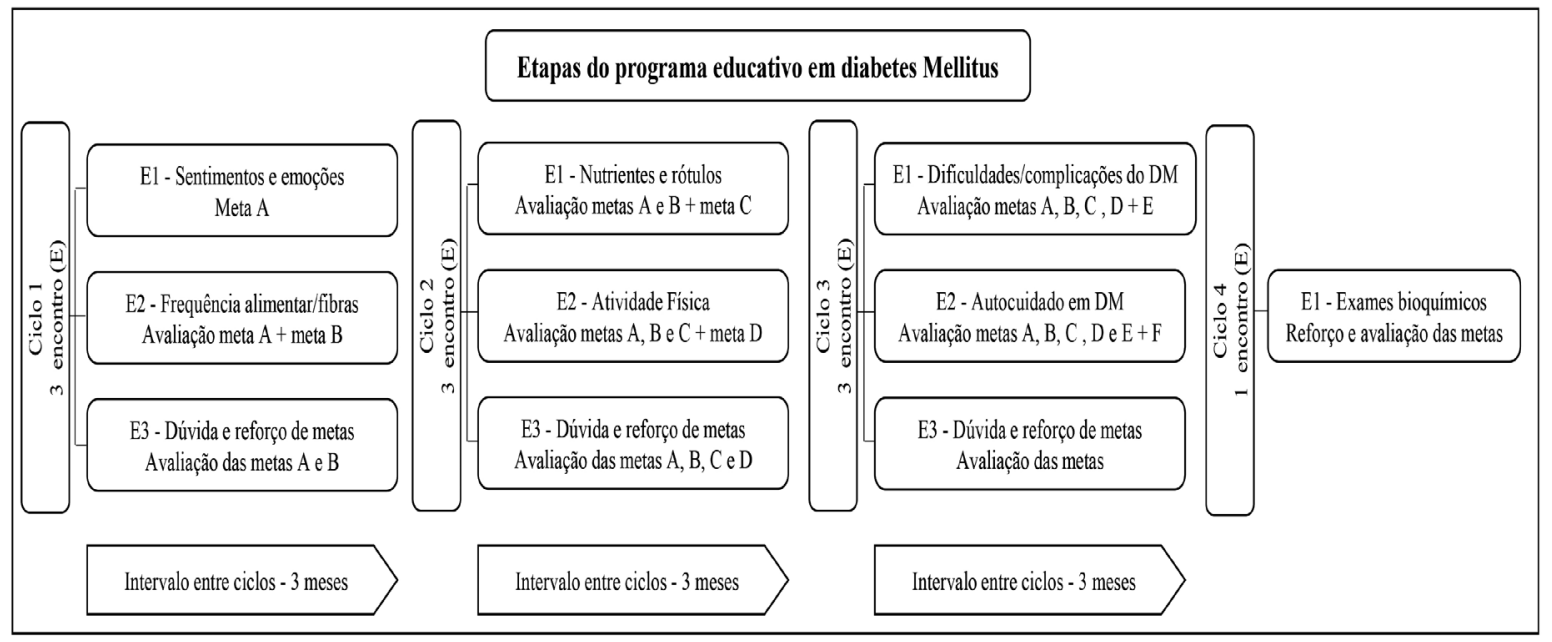

Escala de Empoderamento (DES-SF): permite uma avaliação global da autoeficácia psicossocial relacionada ao diabetes. Este instrumento foi desenvolvido primeiramente com 28 itens e posteriormente foi adequada para oito questões e validado versão para o Brasil (18). Assim, os participantes foram convidados a indicar sua própria capacidade de controlar o diabetes de acordo com as oito questões. As pontuações vão de 1 a 5 , e quanto maior o número, maior será o empoderamento para lidar com o diabetes. A escala é dividida em três pontuações, sendo baixa, de 1 a 2,3 pontos; média, de 2,4 a 3,7 pontos; e alta, de 3,8 a 5,0 pontos (18). Para este estudo utilizou-se apenas duas categorias: pontuações baixa e média unidas em uma categoria e uma segunda categoria para a pontuação alta.

Em relação ao cumprimento de metas e apoiados pelo enfermeiro facilitador, as principais metas propostas pelos usuários foram categorizadas da seguinte forma: a) frequência alimentar, como comer de três em três horas; b) diminuir a ingestão de carboidratos, como redução de arroz no almoço e janta; c) inclusão de fibras na alimentação, como troca do arroz e pão pelo integral ou hortaliças folhosas no almoço; d) realizar atividade física, como caminhadas; e) diminuir ingestão de gorduras, como troca do óleo animal por vegetal ou troca do leite integral pelo desnatado; f) substituição do açúcar pelo adoçante; g) diminuir ou interromper a ingestão de bebidas alcoólicas; h) ou mesmo aumentar algumas destas ações já praticadas. O próprio usuário se autoavaliou quanto à realização das metas e para a análise foi dividida em duas categorias: cumpriu totalmente e cumpriu parcialmente.

Os dados foram organizados em planilha do MSExcel 2013 e analisados com apoio do programa Statistical Package for the Social Sciences $^{\varpi}$ (SPSS), versão 20,0.

A comparação das medianas da HbAlc ao início e no final do estudo realizou-se com o Teste de Wilcoxon. Foi considerado o nível de significância de 5\%. A comparação de proporções das categorias de cumprimento de metas dentro dos grupos com pontuação alta e baixa/média no DES-SF foi realizada por meio do teste Qui-Quadrado de Pearson. A estatística usada como medida de associação 
foi o Odds Ratio (OR).

A pesquisa obteve aprovação do Comitê de Ética em Pesquisa da Universidade Federal de Minas Gerais, Brasil, sob protocolo ${ }^{\circ}$ 426.968/2013, respeitando as normas nacionais e internacionais de ética em pesquisa envolvendo seres humanos como assinatura do Termo de Consentimento Livre e Esclarecido, direito de desistir em participar da pesquisa em qualquer momento salvaguardando o sigilo, ter acesso irrestrito e continuado do cuidado já realizado na Unidade de Saúde da qual faz parte.

\section{RESULTADOS}

Perfil dos usuários: A média de idade dos participantes do estudo foi de 58 anos (DP 9,2 ), e variou entre 34 e 76 anos, com predominância de participantes do sexo feminino $(66,9 \%, 85$ participantes), com relação à escolaridade, $70,9 \%$ (90) possuíam até o ensino fundamental incompleto. Os participantes apresentaram média de 6,4 anos de diagnóstico com Diabetes Mellitus (DP 4). Os resultados evidenciaram também quantitativo relevante de ocorrências de outras doenças como hipertensão arterial, cardiopatia e outros problemas de saúde tais como: hipotireoidismo, reumatismo, esquistossomose e aneurisma (Tabela 1). Em relação ao tipo de tratamento realizado, 4\% (5) tomavam apenas insulina, 22\% (28) tomavam insulina e hipoglicemiante oral e 74\% (94) tomavam apenas hipoglicemiante oral.

Em relação à escala de empoderamento para lidar com o Diabetes Mellitus, 61,4\% (78) foram classificados como alta capacidade, $37,8 \%$ (48) como média e $0,8 \%$ (1) como baixa capacidade. Sobre o cumprimento de metas, 33,9\% (43) dos usuários auto declararam que cumpriram parcialmente e $66,1 \%$ (84) cumpriram totalmente.

Para todos os 127 participantes do estudo, a média da HbAlc antes da intervenção era de $8,1 \%$ (DP 2,0) e ao final do programa de 7,5\% (DP 1,7). Em função da distribuição não normal e comparação entre as medianas da HbAlc, o teste de Wilcoxon apresentou $\mathrm{p}<0,001$, sendo que a mediana da HbAlc

Tabela 1. Distribuição das variáveis sociodemográficas e clínicas dos usuários com Diabetes Mellitus tipo $2(\mathrm{n}=127)$.

\begin{tabular}{|c|c|c|}
\hline \multirow{2}{*}{$\begin{array}{l}\text { Variável } \\
\text { Idade (Média DP) }\end{array}$} & \multicolumn{2}{|c|}{ Média DP ou n \% } \\
\hline & 58 & 9,2 \\
\hline Tempo de diagnostico (Média DP) & 6,4 & 4 \\
\hline \multicolumn{3}{|l|}{ Sexo $(n \%)$} \\
\hline Feminino & 85 & 66,9 \\
\hline \multicolumn{3}{|l|}{ Escolaridade (n \%) } \\
\hline$\leq$ até três anos de estudo & 90 & 70,9 \\
\hline \multicolumn{3}{|l|}{ Estado Civil (n \%) } \\
\hline Com Companheiro & 94 & 74 \\
\hline \multicolumn{3}{|l|}{ Ocupação (n \%) } \\
\hline Aposentado & 72 & 56,7 \\
\hline \multicolumn{3}{|l|}{ Comorbidades (n \%) } \\
\hline Hipertensão arterial & 111 & 87,4 \\
\hline
\end{tabular}


antes da intervenção era de 7,7\% (Mín.: 5,3; Máx.: 13,5) e após a intervenção 7,1\% (Mín.: 5,2; Máx.: 13,7).

Empoderamento e cumprimento de metas: Realizou-se teste de associação entre os grupos de pontuação na escala de empoderamento e o cumprimento de metas (total ou parcial) dos participantes. Foi observado que a chance de cumprimento total de metas no grupo com maior pontuação em empoderamento é 2,98 vezes mais que no grupo com menor pontuação em empoderamento (IC 95\%: 1,30-6,86) (Tabela 2).

Tabela 2. Comparação entre empoderamento e cumprimento de metas do autocuidado dos usuários com Diabetes Mellitus tipo 2.

\begin{tabular}{lcccc}
\hline Variáveis & $\begin{array}{c}\text { Cumpriu totalmente } \\
\mathbf{N}(\%)\end{array}$ & $\begin{array}{c}\text { Cumpriu parcialmente } \\
\mathbf{N}(\%)\end{array}$ & OR (IC 95\%) & $p^{*}$ \\
\hline DES final & & & & \\
Alto & $59(75,6)$ & $19(24,4)$ & $2,98(1,30-6,86)$ & 0,004 \\
Baixo e médio & $25(51,0)$ & $24(49,0)$ & 1 & \\
\hline
\end{tabular}

*Teste de Qui-quadrado de Pearson, DES-SF: escala de empoderamento.

\section{DISCUSSÃO E CONCLUSÃO}

A média de idade dos participantes foi de 58 anos, o que caracteriza um grupo considerado jovem em comparação com outros estudos, que apresentam participantes com média de idade acima de 60 anos $(8,12)$. $\mathrm{O}$ tempo desde o diagnóstico é relativamente baixo, observando-se a média dos resultados dos participantes do estudo. O principal viés deste dado é o de memória, visto que é determinado pelo fato de ser uma informação auto-referida. De toda forma, sabe-se que o usuário já apresenta de cinco a dez anos de instalação do diabetes quando o diagnóstico ocorre $(13,14)$.

A média geral da HbAlc como controle metabólico foi relativamente baixa, visto que se espera o controle ideal abaixo de $7 \%$ e aceita-se um nível de $8 \%$ em idosos já fragilizados (pessoas em que os riscos do controle glicêmico intensivo são maiores do que os benefícios potenciais) $(13,14)$. Tanto o usuário com HbAlc controlada quanto al- terada necessita propor metas de mudanças de comportamento e cumpri-las para seguir seu tratamento. Destaca-se que o programa educativo permitiu auxiliar o usuário nas tomadas de decisões adequadas para cumprir metas e desenvolver comportamentos de autocuidado. Por esta razão, reforça a importância do cuidado da enfermagem ao acompanhar o desempenho do usuário na realização das metas, bem como prever fatores que interferem no seu cumprimento e autocuidado para o controle do diabetes (11, 19). Corroborando evidências da literatura, o cumprimento de metas reflete do apoio profissional e suporte em torno do usuário que são importantes para o seu autocuidado com a alimentação, exercício físico e controle do peso, melhorando seu manejo continuamente com o diabetes $(8,19,20)$.

Em relação aos tipos de metas propostas, a alimentação foi o principal alvo na mudança de comportamento dos usuários, no que tange à frequência, quantidade e inclusão de fibras. A mudança dos hábitos alimentares é um dos cuidados essenciais para o controle 
do diabetes e deve ser pautada na seleção dos alimentos e no fracionamento das refeições (21). Essas mudanças, se colocadas em prática, melhoram a resistência à insulina e diminuem os níveis da glicose plasmática (8).

A participação do usuário na tomada de decisões e reconhecimento das principais mudanças pode ser a chave para que ele desempenhe as práticas de autocuidado para o diabetes. É importante distinguir que não é possível generalizar o que se espera dos usuários, visto que apresentam níveis de compreensão diferentes, com níveis de escolaridade diversificados. Segundo autores, existe relação entre o cumprimento das atividades de autocuidado e as características sociodemográficas e clínicas $(20,22)$. Nesse sentido, ao invés de classificar o usuário somente como aderente ou não às práticas de autocuidado, deve-se avaliar o contexto no qual os comportamentos acontecem (23).

Em relação à aplicação do protocolo para mudança de comportamento, foi identificado que todos os usuários propuseram metas que contribuiriam para o controle do diabetes, sendo que todos conseguiram cumpri-las total ou parcialmente durante o programa educativo. Este resultado evidencia que os participantes reconheceram suas responsabilidades frente ao gerenciamento do autocuidado e se comprometeram com mudanças que pudessem ajudá-los no cuidado com a própria saúde (24). Estudos que utilizaram o mesmo protocolo também demonstraram resultados favoráveis quanto ao desenvolvimento de metas no contexto do diabetes, possibilitando ao usuário melhor adesão e empoderamento para as práticas de autocuidado $(4,5)$.

Os usuários que estavam com maior pontuação para o empoderamento apresentaram maior tendência ao cumprimento total de metas. De alguma forma, esta associação mostra que o empoderamento para o autocuidado pode ser uma ferramenta potencial para o controle do diabetes $(8,9)$. Corroborando evidências da literatura, estudos experimentais encontraram resultados positivos quanto ao cumprimento de metas ao utilizar programa educativo na abordagem do empoderamento para melhorar as práticas de autocuidado $(7,11)$. Espera-se que o autocuidado implantado, principalmente precocemente após o diagnóstico, diminua o aparecimento de complicações. De forma oposta, estudo afirma que não é necessário desenvolver o empoderamento em usuários com pouco tempo de diagnóstico, em função de adquirirem o controle metabólico apenas com o uso de medicamentos (12).

Dentro do ambiente da Atenção Primária à Saúde, o enfermeiro, em seu inerente papel de educador, pode desenvolver programas educativos na abordagem do empoderamento a fim de oferecer ao usuário condições para gerir de maneira eficaz a sua saúde (24). Este profissional da saúde está mencionarmais presente junto aos usuários e possui competências de promover acolhimento e construir vínculos para a continuidade de práticas educativas (14).

O enfermeiro é o agente potencial de mudança e que pode ajudar o usuário a resolver problemas diários para melhorar sua capacidade de tomar decisões informadas e desenvolver habilidades para aprimorar a capacidade para cuidar da própria saúde (11, 19). Portanto, os resultados evidenciam, juntamente com dados da literatura, que este profissional pode enriquecer e aprimorar sua prática assistencial ao utilizar estratégias educativas na abordagem do empoderamento a fim de proporcionar ao usuário o desenvolvimento de metas para o controle do Diabetes Mellitus tipo 2 (6).

Algumas limitações são apontadas no estudo, tais como: inexistência de grupo controle, que limita o estabelecimento de evidências de associação com relação à intervenção avaliada; e os pacientes que participaram do estudo poderiam ser aqueles que estavam mais motivados e proativos na busca de apoio para controlar o diabetes.

Com os resultados do estudo, sugere-se 
novas abordagens e com desenhos metodológicos diferentes que possam imergir no entendimento da subjetividade do empoderamento, além de maximizar pesquisas longitudinais e de maior duração que trabalhem com o autocuidado do diabetes com vistas à diminuição de suas complicações.

Evidenciou-se que o programa educativo baseado no empoderamento das práticas de autocuidado favoreceu o cumprimento de metas em usuários com diabetes tipo 2 para na Atenção Primária à Saúde. O grupo de usuários participantes da intervenção conduzida pelo enfermeiro apresentou como destaque a associação entre estar empoderado para mudança de comportamento e o cumprimento de metas. O enfermeiro, como um dos membros da equipe da Atenção Primária à Saúde, atua de maneira contínua na prevenção e promoção da saúde em diabetes tipo 2. Com isso, é importante que a assistência da enfermagem seja baseada numa escuta qualificada e diálogo problematizador, para acompanhar o desempenho do usuário na realização e cumprimento de metas a fim de proporcionar o controle do Diabetes Mellitus. Estudos com enfoque na educação de comportamentos com a saúde, reforçam a necessidade de enfermeiros aprimorar suas práticas e buscar estratégias que auxilie o usuário a ser agente de transformação da sua realidade.

\section{REFERÊNCIAS}

1. International Diabetes Federation (IDF). Diabetes Atlas. Eighth edition [Internet]. IDF; 2017 [citado 08 jan 2018]. 147 p. Disponível em: http://www.diabetesatlas. org/

2. Li L, Ji L, Guo X, Ji Q, Gu W, Zhi X, et al. Prevalence of microvascular diseases among tertiary care Chinese with early versus late onset of type 2 diabetes. J Diabetes Complications. 2015; 29(1): 32-7.
3. American Diabetes Association (ADA). Standards of medical care in diabetes - 2017. Diabetes Care. 2017; 40 (sup 1) (January): s6-10.

4. Anderson RM, Funnell MM, Aikens JE, Krein SL, Fitzgerald JT, Nwankwo R et al. Evaluating the Efficacy of an Empowerment-Based Self-Management Consultant Intervention: Results of a Two-Year Randomized Controlled Trial. Ther Patient Educ. 2009; 1(1): 3-11.

5. Chen L, Pei JH, Kuang J, Chen HM, Chen Z, Li ZW et al. Effect of lifestyle intervention in patients with type 2 diabetes: A meta-analysis. Metabolism. 2015; 64(2): 338-47.

6. Rossi MC, Lucisano G, Funnell M, Pintaudi B, Bulotta A, Gentile S et al. Interplay among patient empowerment and clinical and person-centered outcomes in type 2 diabetes. The BENCH-D study. Patient Educ Couns. 2015; 98(9): 1142-9.

7. Tol A, Alhani F, Shojaeazadeh D, Sharifirad G, Moazam N. An empowering approach to promote the quality of life and self-management among type 2 diabetic patients. J Educ Health Promot. 2015; 4:13.

8. Chen MF, Wang RH, Lin KC, Hsu HY, Chen SW. Efficacy of an empowerment program for Taiwanese patients with type 2 diabetes: A randomized controlled trial. Appl Nurs Res. 2015; 28(4): 366-73.

9. Wong CK, Wong WC, Lam CL, Wan YF, Wong WH, Chung KL et al. Effects of patient empowerment programme (PEP) on clinical outcomes and health service utilization in type 2 diabetes mellitus in primary care: An observational matched cohort study. PLoS One [Internet]. 2014 [citado 10 jul 2017]; 9(5): e95328. Disponível em: http://journals.plos.org/ plosone/article?id=10.1371/journal. pone.0095328

10. Chaves FA. Tradução e Adaptação Transcultural do "Behavior Change Protocol" para a língua portuguesa-Brasil. [Disser- 
tação de Mestrado]: Belo Horizonte: Universidade Federal de Minas Gerais; 2014. $110 \mathrm{p}$.

11. Souza DAS, Reis IA, Cortez DN, Afonso G de S, Torres H de C. Avaliação da visita domiciliar para o empoderamento do autocuidado em diabetes. Acta Paul Enferm. 2017; 30(4): 350-7.

12. Fitzgerald M, O'Tuathaigh C, Moran J. Investigation of the relationship between patient empowerment and glycaemic control in patients with type 2 diabetes: a cross-sectional analysis. BMJ Open [Internet]. 2015 [citado 10 jul 2017]; 5(12): e008422. Disponível em: https://www. ncbi.nlm.nih.gov/pubmed/26685021

13. American Diabetes Association (ADA). Standard of medical care in diabetes 2017. Diabetes Care. 2017;40 (sup 1)(January): s33-43.

14. Sociedade Brasileira de Diabetes. Diretrizes Sociedade Brasileira de Diabetes 2017-2018 [Internet]. São Paulo: Editora Clannad; 2017 [citado 10 abr 2018]. 383 p. Disponível em: http://www.diabetes. org.br/profissionais/images/2017/diretrizes/diretrizes-sbd-2017-2018.pdf

15. Dutra HS, Reis VN. Desenhos de Estudos Experimentais e Quase-Experimentais: Definições e Desafios na Pesquisa em Enfermagem. Rev enferm UFPE on line [Internet]. 2016 [citado 10 jul 2017]; 10(6): 2230-41. Disponível em: https://periodicos.ufpe.br/revistas/revistaenfermagem/ article/view/11238/12841

16. Cortez DN, Reis IA, Souza DA, Macedo MM, Torres HC. Complications and the time of diagnosis of diabetes mellitus in primary care. Acta Paul Enferm. 2015; 28(3): 250-5.

17. Campbell MK, Thomson S, Ramsay CR, MacLennan GS, Grimshaw JM. Sample size calculator for cluster randomized trials. Comput Biol Med. 2004; 34(2): 11325.

18. Chaves FF, Reis IA, Pagano A, Torres HC. Tradução, adaptação cultural e validação do Diabetes Empowerment Scale-Short Form. Rev Saude Publica. 2017; 51: 1-9. 19. Arda Sürücü H, Büyükkaya Besen D. Predictors of Empowerment in Individuals with Type 2 Diabetes Mellitus. J Transcult Nurs [Internet]. 2017 [citado 10 jul 2017]:104365961775025. Disponível em: http://journals.sagepub. com/doi/10.1177/1043659617750259

20. Genz J, Haastert B, Müller H, Verheyen F, Cole D, Rathmann W et al. Socioeconomic factors and effect of evidence-based patient information about primary prevention of type 2 diabetes mellitus - are there interactions? BMC Res Notes [Internet]. 2014 [citado 10 jul 2017]; 7(1): 541. Disponível em: https://www.ncbi. nlm.nih.gov/pmc/articles/PMC4148532/ pdf/13104_2014_Article_3076.pdf

21. Shrivastava SR, Shrivastava PS, Ramasamy J. Role of self-care in management of diabetes mellitus. J Diabetes Metab Disord [Internet]. 2013 [citado 10 jul 2017]; 12(1): 14. Disponível em: https:// jdmdonline.biomedcentral.com/track/ pdf/10.1186/2251-6581-12-14

22. Clark ML, Utz SW. Social determinants of type 2 diabetes and health in the United States. World J Diabetes [Internet]. 2014 [citado 10 jul 2017]; 5(3): 296-304. Disponível em: https://www.wjgnet. com/1948-9358/full/v5/i3/296.htm

23. Rezende Neta DS, Silva ARV, Silva GRF. Adesão das pessoas com diabetes mellitus ao autocuidado com os pés. Rev. Bras. Enferm. [Internet]. 2015 [citado 10 jul 2017]; 68(1): 1116. Disponível em: http://www.scielo. $\mathrm{br} /$ scielo.php?script=sci_arttext\&pi$\mathrm{d}=$ S0034-71672015000100111\&lng=en.

24. Torres HC. O empoderamento nas práticas educativas orientadas à autonomia no cuidado em saúde. In: Torres $\mathrm{H}$ de C, Reis IA, Pagano AS, editors. Empoderamento do pesquisador nas ciências da saúde. 1st ed. Belo Horizonte: FALE/UFMG; 2015. p. 1-18. 